Article

\title{
Statistical Solitons and Inequalities for Statistical Warped Product Submanifolds
}

\author{
Aliya Naaz Siddiqui ${ }^{1}$, Bang-Yen Chen ${ }^{2, *}$ (1) and Oguzhan Bahadir ${ }^{3}$ \\ 1 Department of Mathematics, Faculty of Natural Sciences, Jamia Millia Islamia, New Delhi 110025, India \\ 2 Department of Mathematics, Michigan State University, 619 Red Cedar Road, \\ East Lansing, MI 48824-1027, USA \\ 3 Department of Mathematics, Faculty of Science and Letters, Kahramanmaras Sutcu Imam University, \\ Kahrmanmaras 46100, Turkey \\ * Correspondence: chenb@msu.edu
}

Received: 25 July 2019; Accepted: 26 August 2019; Published: 1 September 2019

\begin{abstract}
Warped products play crucial roles in differential geometry, as well as in mathematical physics, especially in general relativity. In this article, first we define and study statistical solitons on Ricci-symmetric statistical warped products $\mathbb{R} \times_{\mathfrak{f}} N_{2}$ and $N_{1} \times_{\mathfrak{f}} \mathbb{R}$. Second, we study statistical warped products as submanifolds of statistical manifolds. For statistical warped products statistically immersed in a statistical manifold of constant curvature, we prove Chen's inequality involving scalar curvature, the squared mean curvature, and the Laplacian of warping function (with respect to the Levi-Civita connection). At the end, we establish a relationship between the scalar curvature and the Casorati curvatures in terms of the Laplacian of the warping function for statistical warped product submanifolds in the same ambient space.
\end{abstract}

Keywords: statistical warped product submanifold; statistical manifold; B.Y.Chen inequality; Casorati curvatures; statistical soliton

MSC: 53B30; 53C15; 53C25

\section{Introduction}

Statistical manifolds were introduced in 1985 by S. Amari [1] in terms of information geometry, and they were applied by Lauritzen in [2]. Such manifolds have an important role in statistics as the statistical model often forms a geometrical manifold.

Let $\tilde{\nabla}$ be an affine connection on a (pseudo-)Riemannian manifold $(\tilde{N}, \tilde{g})$. The affine connection $\tilde{\nabla}^{*}$ on $\tilde{N}$ satisfying:

$$
E \tilde{g}(F, G)=\tilde{g}\left(\tilde{\nabla}_{E} F, G\right)+\tilde{g}\left(F, \tilde{\nabla}_{E}^{*} G\right), \quad \forall E, F, G \in \Gamma(T \tilde{N}),
$$

is called a dual connection of $\tilde{\nabla}$ with respect to $\tilde{g}$.

The triplet $(\tilde{N}, \tilde{\nabla}, \tilde{g})$ is called a statistical manifold if:

(a) the Codazzi equation $\left(\tilde{\nabla}_{E} \tilde{g}\right)(F, G)=\left(\tilde{\nabla}_{F} \tilde{g}\right)(E, G)$ holds, for any $E, F, G \in \Gamma(T \tilde{N})$;

(b) the torsion tensor field of $\tilde{\nabla}$ vanishes.

If $(\tilde{\nabla}, \tilde{g})$ is a statistical structure on $\tilde{N}$, then $\left(\tilde{\nabla}^{*}, \tilde{g}\right)$ is also a statistical structure. The connections $\tilde{\nabla}$ and $\tilde{\nabla}^{*}$ satisfy $\left(\tilde{\nabla}^{*}\right)^{*}=\tilde{\nabla}$. On the other hand, we have $\tilde{\nabla}^{0}=\frac{1}{2}\left(\tilde{\nabla}+\tilde{\nabla}^{*}\right)$, where $\tilde{\nabla}^{0}$ is the Levi-Civita connection of $\tilde{N}$.

One of the most fruitful generalizations of Riemannian products is the warped product defined in [3]. The notion of warped products plays very important roles in differential geometry and in 
mathematical physics, especially in general relativity. For instance, space-time models in general relativity are usually expressed in terms of warped products (cf., e.g., [4,5]).

In 2006, L. Todjihounde [6] defined a suitable dualistic structure on warped product manifolds. Furthermore, Furuhata et al. [7] defined Kenmotsu statistical manifolds and studied how to construct such structures on the warped product of a holomorphic statistical manifold [8] and a line. In [9], H. Aytimur and C. Ozgur studied Einstein statistical warped product manifolds. Further, C. Murathan and B. Sahin [10] studied and obtained the Wintgen-like inequality for statistical submanifolds of statistical warped product manifolds.

The Ricci solitons are special solutions of the Ricci flow of the Hamilton. In Section 4, we define statistical solitons and study the problem under what conditions the base manifold or fiber manifold of a statistical warped product manifold is a statistical soliton.

Curvature invariants play the most fundamental and natural roles in Riemannian geometry. A fundamental problem in the theory of Riemannian submanifolds is (cf. [11]):

Problem A."Establish simple optimal relationships between the main intrinsic invariants and the main extrinsic invariants of a submanifold."

The first solutions of this problem for warped product submanifolds were given in [11,12]. In Section 5, we study this fundamental problem for statistical warped product submanifolds in any statistical manifolds of constant curvature. Our solution to this problem given in this section is derived via the fundamental equations of statistical submanifolds.

An extrinsic curvature of a Riemannian submanifold was defined by Casorati in [13], as the normalized square of the length of the second fundamental form. Casorati curvature has nice applications in computer vision. It was preferred by Casorati over the traditional curvature since it corresponds better to the common intuition of curvature.

Several sharp inequalities between extrinsic and intrinsic curvatures for different submanifolds in real, complex, and quaternionic space forms endowed with various connections have been obtained (e.g., [14-21]). Such inequalities with a pair of conjugate affine connections involving the normalized scalar curvature of statistical submanifolds in different ambient spaces were obtained in [22-26].

Inspired by historical development on the classifications of Casorati curvatures and Ricci curvatures, we establish in Section 6 an inequality for statistical warped product submanifolds in a statistical manifold of constant curvature. In the last section, we provide two examples of statistical warped product submanifolds in the same environment.

\section{Preliminaries}

Let $(\tilde{N}, \tilde{\nabla}, \tilde{g})$ be a statistical manifold and $N$ be a submanifold of $\tilde{N}$. Then, $(N, \nabla, g)$ is also a statistical manifold with the statistical structure $(\nabla, g)$ on $N$ induced from $(\tilde{\nabla}, \tilde{g})$, and we call $(N, \nabla, g)$ a statistical submanifold.

The fundamental equations in the geometry of Riemannian submanifolds are the Gauss and Weingarten formulae and the equations of Gauss, Codazzi, and Ricci (cf. [4,5,27]). In the statistical setting, the Gauss and Weingarten formulae are defined respectively by [28]:

$$
\left.\begin{array}{lr}
\tilde{\nabla}_{E} F=\nabla_{E} F+h(E, F), \quad \tilde{\nabla}_{E}^{*} F=\nabla_{E}^{*} F+h^{*}(E, F), \\
\tilde{\nabla}_{E} \xi=-A_{\tilde{\zeta}}(E)+\nabla_{E}^{\perp} \xi, \quad \tilde{\nabla}_{E}^{*} \xi=-A_{\tilde{\zeta}}^{*}(E)+\nabla_{E}^{\perp *} \xi,
\end{array}\right\}
$$

for any $E, F \in \Gamma(T N)$ and $\xi \in \Gamma\left(T^{\perp} N\right)$, where $\tilde{\nabla}$ and $\tilde{\nabla}^{*}$ (resp., $\nabla$ and $\nabla^{*}$ ) are the dual connections on $\tilde{N}$ (resp., on $N)$. 
The symmetric and bilinear imbedding curvature tensor of $N$ in $\tilde{N}$ with respect to $\tilde{\nabla}$ and $\tilde{\nabla}^{*}$ is denoted as $h$ and $h^{*}$, respectively. The relation between $h\left(\right.$ resp. $h^{*}$ ) and $A_{\tilde{\xi}}$ (resp. $A_{\tilde{\xi}}^{*}$ ) is defined by [28]:

$$
\left.\begin{array}{r}
\tilde{g}(h(E, F), \xi)=g\left(A_{\mathcal{\zeta}}^{*} E, F\right), \\
\tilde{g}\left(h^{*}(E, F), \xi\right)=g\left(A_{\tilde{\zeta}} E, F\right),
\end{array}\right\}
$$

for any $E, F \in \Gamma(T N)$ and $\xi \in \Gamma\left(T^{\perp} N\right)$.

Let $\tilde{R}$ and $R$ be the curvature tensor fields of $\tilde{\nabla}$ and $\nabla$, respectively. The corresponding Gauss, Codazzi, and Ricci equations are given by [28]:

$$
\begin{aligned}
& \tilde{g}(\tilde{R}(E, F) G, H)= g(R(E, F) G, H)+\tilde{g}\left(h(E, G), h^{*}(F, H)\right) \\
&-\tilde{g}\left(h^{*}(E, H), h(F, G)\right), \\
&(\tilde{R}(E, F) G)^{\perp}= \nabla_{E}^{\perp} h(F, G)-h\left(\nabla_{E} F, G\right)-h\left(F, \nabla_{E} G\right) \\
&-\left\{\nabla_{F}^{\perp} h(E, G)-h\left(\nabla_{F} E, G\right)-h\left(E, \nabla_{F} G\right)\right\}, \\
& \tilde{g}\left(\tilde{R}^{\perp}(E, F) \xi, \eta\right)=\tilde{g}(R(E, F) \xi, \eta)+g\left(\left[A_{\zeta}^{*}, A_{\eta}\right] E, F\right),
\end{aligned}
$$

for any $E, F, G, H \in \Gamma(T N)$ and $\xi, \eta \in \Gamma\left(T^{\perp} N\right)$, where $R^{\perp}$ is the Riemannian curvature tensor on $T^{\perp} N$.

Similarly, $\tilde{R}^{*}$ and $R^{*}$ are respectively the curvature tensor fields with respect to $\tilde{\nabla}^{*}$ and $\nabla^{*}$. We can obtain the duals of all Equations (3)-(5) with respect to $\tilde{\nabla}^{*}$ and $\nabla^{*}$. Furthermore,

$$
\tilde{S}=\frac{1}{2}\left(\tilde{R}+\tilde{R}^{*}\right) \text { and } S=\frac{1}{2}\left(R+R^{*}\right)
$$

are respectively the curvature tensor fields of $\tilde{N}$ and $N$ given by [7]. Thus, the sectional curvature $\mathbb{K}^{\nabla, \nabla^{*}}$ on $N$ of $\tilde{N}$ is defined by $[29,30]$ :

$$
\begin{aligned}
\mathbb{K}^{\nabla, \nabla^{*}}(E \wedge F) & =g(S(E, F) F, E) \\
& =\frac{1}{2}\left(g(R(E, F) F, E)+g\left(R^{*}(E, F) F, E\right)\right),
\end{aligned}
$$

for any orthonormal vectors $E, F \in T_{p} N, p \in N$.

Suppose that $\operatorname{dim}(N)=m$ and $\operatorname{dim}(\tilde{N})=n$. Let $\left\{e_{1}, \ldots, e_{m}\right\}$ and $\left\{e_{m+1}, \ldots, e_{n}\right\}$ be respectively the orthonormal basis of $T_{p} N$ and $T_{p}^{\perp} N$ for $p \in N$. Then, the scalar curvature $\sigma^{\nabla, \nabla^{*}}$ of $N$ is given by:

$$
\sigma^{\nabla, \nabla^{*}}=\sum_{1 \leq i<j \leq m} \mathbb{K}^{\nabla, \nabla^{*}}\left(e_{i} \wedge e_{j}\right)
$$

The normalized scalar curvature $\rho$ of $N$ is defined as:

$$
\rho^{\nabla, \nabla^{*}}=\frac{2 \sigma^{\nabla, \nabla^{*}}}{m(m-1)}
$$

The mean curvature vectors $\mathcal{H}$ and $\mathcal{H}^{*}$ of $N$ in $\tilde{N}$ are:

$$
\mathcal{H}=\frac{1}{m} \sum_{i=1}^{m} h\left(e_{i}, e_{i}\right), \quad \mathcal{H}^{*}=\frac{1}{m} \sum_{i=1}^{m} h^{*}\left(e_{i}, e_{i}\right)
$$


Furthermore, we set:

$$
h_{i j}^{a}=\tilde{g}\left(h\left(e_{i}, e_{j}\right), e_{a}\right), h_{i j}^{* a}=\tilde{g}\left(h^{*}\left(e_{i}, e_{j}\right), e_{a}\right),
$$

for $i, j \in\{1, \ldots, m\}, a \in\{m+1, \ldots, n\}$.

A statistical manifold $(\tilde{N}, \tilde{\nabla}, \tilde{g})$ is said to be of constant curvature $\tilde{c} \in \mathbb{R}$, denoted by $\tilde{N}(\tilde{c})$, if the following curvature equation holds:

$$
\tilde{S}(E, F) G=\tilde{c}(g(F, G) E-g(E, G) F), \quad \forall E, F, G \in \Gamma(T \tilde{N}) .
$$

\section{Basics on Statistical Warped Product Manifolds}

Definition 1. [3] Let $\left(N_{1}, g_{1}\right)$ and $\left(N_{2}, g_{2}\right)$ be two (pseudo)-Riemannian manifolds and $\mathfrak{f}>0$ be a differentiable function on $N_{1}$. Consider the natural projections $\pi: N_{1} \times N_{2} \rightarrow N_{1}$ and $\pi^{\prime}: N_{1} \times N_{2} \rightarrow N_{2}$. Then, the warped product $N=N_{1} \times_{\mathfrak{f}} N_{2}$ with warping function $\mathfrak{f}$ is the product manifold $N_{1} \times N_{2}$ equipped with the Riemannian structure such that:

$$
\tilde{g}(E, F)=g_{1}\left(\pi_{*} E, \pi_{*} F\right)+\mathfrak{f}^{2}(u) g_{2}\left(\pi_{*}^{\prime} E, \pi_{*}^{\prime} F\right),
$$

for $E, F \in \Gamma\left(T_{(u, v)} N\right), u \in N_{1}$, and $v \in N_{2}$, where $*$ denotes the tangent map.

Let $\chi\left(N_{1}\right)$ and $\chi\left(N_{2}\right)$ be the set of all vector fields on $N_{1} \times N_{2}$, which is the horizontal lift of a vector field on $N_{1}$ and the vertical lift of a vector field on $N_{2}$, respectively. We have $T\left(N_{1} \times N_{2}\right)=\chi\left(N_{1}\right) \oplus \chi\left(N_{2}\right)$. Thus, it can be seen that $\pi_{*}\left(\chi\left(N_{1}\right)\right)=\Gamma\left(T N_{1}\right)$ and $\pi_{*}^{\prime}\left(\chi\left(N_{2}\right)\right)=\Gamma\left(T N_{2}\right)$. Therefore, $\pi_{*}(X)=E_{1} \in \Gamma\left(T N_{1}\right), \pi_{*}(Y)=F_{1} \in \Gamma\left(T N_{1}\right), \pi_{*}^{\prime}(U)=E_{2} \in$ $\Gamma\left(T N_{2}\right)$ and $\pi_{*}^{\prime}(V)=F_{2} \in \Gamma\left(T N_{2}\right)$, for any $X, Y \in \chi\left(N_{1}\right)$ and $U, V \in \chi\left(N_{2}\right)$.

Recall the following general result from [6] for a dualistic structure on the warped product manifold $N_{1} \times_{\mathfrak{f}} N_{2}$.

Proposition 1. Let $\left(g_{1}, \nabla^{N_{1}}, \nabla^{N_{1} *}\right)$ and $\left(g_{2}, \nabla^{N_{2}}, \nabla^{N_{2} *}\right)$ be dualistic structures on $N_{1}$ and $N_{2}$, respectively. For $X, Y \in \chi\left(N_{1}\right)$ and $U, V \in \chi\left(N_{2}\right), D, D^{*}$ on $N_{1} \times N_{2}$ satisfy:

(a) $\quad D_{X} Y=\nabla_{E_{1}}^{N_{1}} F_{1}$

(b) $D_{X} U=D_{U} X=\frac{E_{1} \mathfrak{f}}{\mathfrak{f}} E_{2}$,

(c) $D_{U} V=\nabla_{E_{2}}^{N_{2}} F_{2}-\frac{\tilde{g}(U, V)}{\mathfrak{f}} \operatorname{grad} \mathfrak{f}$,

(d) $D_{X}^{*} Y=\nabla_{E_{1}}^{N_{1} *} F_{1}$,

(e) $D_{X}^{*} U=D_{U}^{*} X=\frac{E_{1} \mathfrak{f}}{\mathfrak{f}} E_{2}$,

(f) $\quad D_{U}^{*} V=\nabla_{E_{2}}^{N_{2} *} F_{2}-\frac{\tilde{g}(U, V)}{\mathfrak{f}} \operatorname{grad} \mathfrak{f}$,

where $\nabla_{E_{1}}^{N_{1}} F_{1}=\pi *\left(D_{X} Y\right), \nabla_{E_{1}}^{N_{1} *} F_{1}=\pi *\left(D_{X}^{*} Y\right), \nabla_{E_{2}}^{N_{2}} F_{2}=\pi_{*}^{\prime}\left(D_{U} V\right)$, and $\nabla_{E_{2}}^{N_{2} *} F_{2}=\pi_{*}^{\prime}\left(D_{U}^{*} V\right)$. Then, $\left(\tilde{g}, D, D^{*}\right)$ is a dualistic structure on $N_{1} \times N_{2}$.

Furthermore, Todjihounde [6] derived the curvature of the statistical warped product $\tilde{N}=N_{1} \times_{\mathfrak{f}}$ $N_{2}$ in terms of the curvature tensors $R_{1}$ and $R_{2}$ of $N_{1}$ and $N_{2}$, respectively, and its warping function $\mathfrak{f}$.

Lemma 1. Let $\left(\tilde{N}=N_{1} \times_{\mathfrak{f}} N_{2}, D, D^{*}, \tilde{g}\right)$ be a statistical warped product manifold. For $X, Y, Z \in \chi\left(N_{1}\right)$ and $U, V, W \in \chi\left(N_{2}\right)$, we have:

(a) $\tilde{R}(X, Y) Z=R_{1}\left(E_{1}, F_{1}\right) G_{1}$,

(b) $\tilde{R}(U, Y) Z=-\mathfrak{f}^{-1} \operatorname{Hess}_{\mathfrak{f}}(Y, Z) U$,

(c) $\tilde{R}(X, Y) W=0$,

(d) $\tilde{R}(U, V) Z=0$, 
(e) $\tilde{R}(X, V) W=-\mathfrak{f}^{-1} \tilde{g}(V, W) D_{X}($ grad $\mathfrak{f})$,

(f) $\tilde{R}(U, V) W=R_{2}\left(E_{2}, F_{2}\right) G_{2}+\|\operatorname{grad} \mathfrak{f}\|^{2}\left[g_{2}(U, W) V-g_{2}(V, W) U\right]$,

where $\tilde{R}$ denotes the curvature tensor field of $\left(\tilde{N}=N_{1} \times_{\mathfrak{f}} N_{2}, D, D^{*}, \tilde{g}\right)$ and $\operatorname{Hess}_{\mathfrak{f}}(X, Y)=X(Y \mathfrak{f})-\left(\nabla_{X}^{N_{1}} Y\right) \mathfrak{f}$ is the Hessian function of $\mathfrak{f}$ with respect to $\nabla^{N_{1}}$.

The next result from [9] provides the Ricci tensor R̃ic of the statistical warped product manifold.

Lemma 2. Let $\left(\tilde{N}=N_{1} \times_{\mathfrak{f}} N_{2}, D, D^{*}, \tilde{g}\right)$ be a statistical warped product manifold. For $X, Y \in \chi\left(N_{1}\right)$ and $U, V \in \chi\left(N_{2}\right)$, we have:

(a) $\tilde{R} i c(X, Y)=\operatorname{Ric}_{1}(X, Y)-\operatorname{dim}\left(N_{2}\right) \mathfrak{f}^{-1} \operatorname{Hess}_{\mathfrak{f}}(X, Y)$,

(b) $\tilde{R} i c(X, V)=0$,

(c) $\tilde{R} i c(U, V)=\operatorname{Ric}_{2}(U, V)-\left[\mathfrak{f}(\Delta \mathfrak{f})+\left(\operatorname{dim}\left(N_{2}\right)-1\right)\|\operatorname{grad} \mathfrak{f}\|^{2}\right] g_{2}(U, V)$,

where Ric ${ }_{1}$ and Ric $_{2}$ are the Ricci tensors of $N_{1}$ and $N_{2}$, respectively, and $\Delta \mathfrak{f}=\operatorname{div}($ grad $\mathfrak{f})$ is the Laplacian of $\mathfrak{f}$ with respect to $D$.

We recall the following result from [31]. This result is useful in some Riemannian problems like the study of the distance between two manifolds, of the extremes of sectional curvature and is applied successfully in the demonstration of the Chen inequality.

Let $(N, g)$ be a Riemannian submanifold of a Riemannian manifold $(\tilde{N}, \tilde{g})$, and let $f: \tilde{N} \rightarrow \mathbb{R}$ be a differentiable function. Let:

$$
\min _{x_{0} \in N} f\left(x_{0}\right)
$$

be the constrained extremum problem.

Theorem 1. If $x \in N$ is the solution of the problem (11), then:

(a) $(\operatorname{grad} f)(x) \in T_{x}^{\perp} N$,

(b) the bilinear form $\Theta: T_{x} N \times T_{x} N \rightarrow \mathbb{R}$,

$$
\Theta(E, F)=\operatorname{Hess}_{f}(E, F)+\tilde{g}\left(h^{\prime}(E, F),(\operatorname{grad} f)(x)\right)
$$

is positive semi-definite, where $h^{\prime}$ is the second fundamental form of $N$ in $\tilde{N}$ and grad $f$ denotes the gradient of $f$.

\section{Statistical Solitons on Statistical Warped Product Manifolds}

The Ricci solitons model the formation of singularities in the Ricci flow, and they correspond to self-similar solutions. R. Hamilton [32] introduced the study of Ricci solitons as fixed or stationary points of the Ricci flow in the space of the metrics on Riemannian manifolds modulo diffeomorphisms and scaling. Since then, many researchers studied Ricci solitons for different reasons and in different ambient spaces (for example [33-35]). A complete Riemannian manifold $(\tilde{N}, \tilde{g})$ is called a Ricci soliton $(\tilde{N}, \tilde{g}, \zeta, \lambda)$ if there exists a smooth vector field $\zeta$ and a constant $\lambda \in \mathbb{R}$ such that:

$$
2 \tilde{R} i c=2 \lambda \tilde{g}-\mathcal{L}_{\zeta} \tilde{g},
$$

where $\mathcal{L}_{\zeta}$ denotes the Lie derivative along $\zeta$ and $\tilde{R} i c$ is the Ricci tensor of $\tilde{g}$.

A generalization of Ricci solitons in the framework of manifolds endowed with an arbitrary linear connection $\tilde{\nabla}$, different from the Levi-Civita connection of $\tilde{g}$, is defined in [36] as follows: 
Let $(\tilde{N}, \tilde{\nabla})$ be a manifold and $\zeta \in \chi(\tilde{N})$. A triple $(\tilde{g}, \zeta, \lambda)$ is called a $\tilde{\nabla}$-Ricci soliton if $\tilde{\nabla} \zeta+\tilde{\mathcal{Q}}+\lambda I=0$ holds, where $\tilde{\mathcal{Q}}$ is the Ricci operator of $\tilde{N}$ defined by $\tilde{g}(\tilde{\mathcal{Q}} E, F)=\tilde{R} i c(E, F)$, for vector fields $E, F$ on $\tilde{N}$.

The statistical manifold $(\tilde{N}, \tilde{\nabla}, \tilde{g})$ is called Ricci-symmetric if the Ricci operator $\tilde{\mathcal{Q}}$ with respect to $\tilde{\nabla}$ (equivalently, the dual operator $\tilde{\mathcal{Q}}^{*}$ with respect to $\tilde{\nabla}^{*}$ ) is symmetric (cf. [36,37]).

Based on these, we have the following.

Definition 2. A pair $(\zeta, \lambda)$ is called a statistical soliton on a Ricci-symmetric statistical manifold $(\tilde{N}, \tilde{\nabla}, \tilde{g})$ if the triple $(\tilde{g}, \zeta, \lambda)$ is $\tilde{\nabla}$-Ricci and $\tilde{\nabla}^{*}$-Ricci solitons, i.e., we have:

$$
\tilde{\nabla} \zeta+\tilde{\mathcal{Q}}+\lambda I=0,
$$

and:

$$
\tilde{\nabla}^{*} \zeta+\tilde{\mathcal{Q}}^{*}+\lambda I=0,
$$

where $\tilde{g}(\tilde{\mathcal{Q}} E, F)=\tilde{R} i c(E, F)$ and $\tilde{g}\left(\tilde{\mathcal{Q}}^{*} E, F\right)=\tilde{R} i c^{*}(E, F)$, for all vector fields on $\tilde{N}$, and $\tilde{R} i c$ and $\tilde{R} i c^{*}$ denote the Ricci tensor fields with respect to $\tilde{\nabla}$ and $\tilde{\nabla}^{*}$, respectively.

The main purpose of this section is to study the problem: under what conditions does the base manifold or fiber manifold of the statistical warped product manifold become a statistical soliton?

Let $\left(N_{1}, \nabla^{N_{1}}, \nabla^{N_{1} *}, g_{1}\right)$ and $\left(N_{2}, \nabla^{N_{2}}, \nabla^{N_{2} *}, g_{2}\right)$ be the Ricci-symmetric statistical manifolds. Denote the Ricci-symmetric statistical warped product manifold by $\left(\tilde{N}=N_{1} \times_{f} N_{2}, D, D^{*}, \tilde{g}=g_{1}+f^{2} g_{2}\right)$. Let $\zeta=\left(\zeta_{1}, \zeta_{2}\right) \in \chi(\tilde{N})$ be a vector field on $\tilde{N}$. Then, the pair $(\zeta, \lambda)$ on $(\tilde{N}, \tilde{\nabla}, \tilde{g})$ is called a statistical soliton if the triple $(\tilde{g}, \zeta, \lambda)$ is both $D$-Ricci and $D^{*}$-Ricci solitons, given by (12) and (13).

It follows from Lemma 2 that the Ricci tensor of $\tilde{N}$ is given as below:

$$
\begin{aligned}
\tilde{R} i c= & R i c_{1}-\mathfrak{f}^{-1} \operatorname{dim}\left(N_{2}\right) \text { Hess }_{\mathfrak{f}}+\text { Ric }_{2} \\
& -\left[\mathfrak{f}(\Delta \mathfrak{f})+\left(\operatorname{dim}\left(N_{2}\right)-1\right) \| \text { grad } \mathfrak{f} \|^{2}\right] g_{2} .
\end{aligned}
$$

Thus, (12) and (13) can be rewritten as:

$$
\begin{gathered}
\nabla^{N_{1}} \zeta_{1}+\nabla^{N_{2}} \zeta_{2}+\text { Ric }_{1}-\mathfrak{f}^{-1} \operatorname{dim}\left(N_{2}\right) \text {Hess }_{\mathfrak{f}}+\operatorname{Ric}_{2} \\
-\left[\mathfrak{f}(\Delta \mathfrak{f})+\left(\operatorname{dim}\left(N_{2}\right)-1\right)\|\operatorname{grad} \mathfrak{f}\|^{2}\right] g_{2}+\lambda g_{1}+\lambda \mathfrak{f}^{2} g_{2}=0,
\end{gathered}
$$

and:

$$
\begin{gathered}
\nabla^{N_{1} *} \zeta_{1}+\nabla^{N_{2} *} \zeta_{2}+R i c_{1}^{*}-\mathfrak{f}^{-1} \operatorname{dim}\left(N_{2}\right) \operatorname{Hess}_{\mathfrak{f}}^{D^{*}}+R i c_{2}^{*} \\
-\left[\mathfrak{f}\left(\Delta^{D^{*}} \mathfrak{f}\right)+\left(\operatorname{dim}\left(N_{2}\right)-1\right)\|\operatorname{grad} \mathfrak{f}\|^{2}\right] g_{2}+\lambda g_{1}+\lambda \mathfrak{f}^{2} g_{2}=0,
\end{gathered}
$$

respectively.

Throughout this section, we use the statistical warped products as Ricci-symmetric.

We give the following results by applying Lemma 2 :

Lemma 3. Let $\left(\tilde{N}=\mathbb{R} \times_{\mathfrak{f}} N_{2}, D, D^{*}, \tilde{g}\right)$ be a statistical warped product manifold, where $\left(\mathbb{R}, \nabla^{\mathbb{R}}, d z^{2}\right)$ is a trivial statistical manifold of dimension one and $\operatorname{dim}\left(N_{2}\right)=k$. Then, for $U, V \in \chi\left(N_{2}\right)$, we have:

(a) $\tilde{R} i c(\partial z, \partial z)=-k \mathfrak{f}^{-1} \ddot{\mathfrak{f}}$,

(b) $\tilde{R} i c(\partial z, V)=0$,

(c) $\tilde{R} i c(U, V)=\operatorname{Ric}_{2}(U, V)-\left[\ddot{\mathfrak{f}}+(k-1) \dot{\mathfrak{f}}^{2}\right] g_{2}(U, V)$. 
Proposition 2. Let $(\zeta, \lambda)$ be a statistical soliton on statistical warped product manifold $\left(\tilde{N}=\mathbb{R} \times_{\mathfrak{f}} N_{2}, D, D^{*}, \tilde{g}=d z^{2}+\mathfrak{f}^{2} g_{2}\right)$ with $\operatorname{dim}(\mathbb{R})=1$ and $\operatorname{dim}\left(N_{2}\right)=k$. Then:

$$
\operatorname{Hess}_{\mathfrak{f}}=\frac{\mathfrak{f} \lambda}{k} .
$$

Proof. Since $\tilde{N}$ is a statistical soliton, then from (6), we have:

$$
\tilde{g}\left(\tilde{\nabla}_{\partial z} \zeta, \partial z\right)+\tilde{R} i c(\partial z, \partial z)+\lambda \tilde{g}(\partial z, \partial z)=0 .
$$

By taking into account Lemma 3 and $\operatorname{Ric}_{1}(\partial z, \partial z)=0$, we get:

$$
-\tilde{g}\left(\zeta, \tilde{\nabla}_{\partial z}^{*} \partial z\right)-k \mathfrak{f}^{-1} \operatorname{Hess}_{\mathfrak{f}}(\partial z, \partial z)+\lambda \tilde{g}(\partial z, \partial z)=0,
$$

which gives $\operatorname{Hess}_{\mathfrak{f}}(\partial z, \partial z)=\left(\frac{\mathfrak{f} \lambda}{k}\right) \tilde{g}(\partial z, \partial z)$.

Theorem 2. Let $\zeta=\left(\partial z, \zeta_{2}\right) \in \chi(\tilde{N})$ be a vector field on statistical warped product manifold $\left(\tilde{N}=\mathbb{R} \times_{\mathfrak{f}} N_{2}, D, D^{*}, \tilde{g}=d z^{2}+f^{2} g_{2}\right)$ with $\operatorname{dim}(\mathbb{R})=1$ and $\operatorname{dim}\left(N_{2}\right)=k$. If $(\zeta, \lambda)$ is a statistical soliton on $\tilde{N}$, then:

(a) $\left(N_{2}, g_{2}, \zeta_{2}, \lambda_{2}\right)$ is a statistical soliton on $\left(N_{2}, \nabla^{N_{2}}, \nabla^{N_{2} *}, g_{2}\right)$, where $\lambda_{2}=(k-1)\left[\ddot{f} \ddot{q} \dot{\mathfrak{f}}^{2}\right]$,

(b) $\mathfrak{f}(z)=a z+b$ if $\lambda=0$,

(c) $\mathfrak{f}(z)=\cosh (a z+b)$ if $\lambda \neq 0$,

where $a, b \in \mathbb{R}$.

Proof. From Equation (15) and Lemma 3, we have:

$$
\begin{aligned}
& \nabla^{N_{1}} \partial z+\nabla^{N_{2}} \zeta_{2}+R i c_{1}-k \mathfrak{f}^{-1} \ddot{\mathfrak{f}}+R i c_{2} \\
& -\left(\ddot{\mathfrak{f}}+(k-1) \dot{\mathfrak{f}}^{2}\right) g_{2}+\lambda g_{1}+\lambda \mathfrak{f}^{2} g_{2}=0 .
\end{aligned}
$$

Note $g_{1}\left(\nabla_{\partial z}^{N_{1}} \partial z, \partial z\right)=0$ and $\operatorname{Ric}_{1}(\partial z, \partial z)=0$. Thus, the above equation becomes:

$$
\nabla^{N_{2}} \zeta_{2}-k \mathfrak{f}^{-1} \ddot{\mathfrak{f}}+R i c_{2}-\left(\ddot{\mathfrak{f}}+(k-1) \dot{\mathfrak{f}}^{2}\right) g_{2}+\lambda g_{1}+\lambda \mathfrak{f}^{2} g_{2}=0,
$$

from which we get:

$$
\begin{gathered}
\lambda=k \mathfrak{f}^{-1} \ddot{\mathfrak{f}}, \\
\nabla^{N_{2}} \zeta_{2}+R i c_{2}+\left[\lambda \mathfrak{f}^{2}-\left(\ddot{\mathfrak{f}}+(k-1) \dot{\mathfrak{f}}^{2}\right)\right] g_{2}=0 .
\end{gathered}
$$

Putting (17) into the Equation (18), we arrive at:

$$
\nabla^{N_{2}} \zeta_{2}+R i c_{2}+(k-1)\left[\ddot{\mathfrak{f}}-\dot{\mathfrak{f}}^{2}\right] g_{2}=0 .
$$

Similarly, by using (16), we derive:

$$
\nabla^{N_{2} *} \zeta_{2}+R i c_{2}^{*}+(k-1)\left[\ddot{\mathfrak{f}}-\dot{\mathfrak{f}}^{2}\right] g_{2}=0 .
$$

Thus, $\left(N_{2}, g_{2}, \zeta_{2},(k-1)\left[\ddot{f} \ddot{f}-\dot{\mathfrak{f}}^{2}\right]\right)$ is a statistical soliton provided that $(k-1)\left[\ddot{\mathfrak{f}}-\dot{\mathfrak{f}}^{2}\right]$ is constant. On the other hand, by using (17), we have the following cases:

(a) if $\lambda=0$, then $\mathfrak{f}(z)=a z+b$, and

(b) if $\lambda \neq 0$, then $\mathfrak{f}(z)=\cosh (a z+b)[9]$, 
where $a, b$ are real constants.

Before proving the next result, we state the following:

Lemma 4. Let $\left(\tilde{N}=N_{1} \times_{\mathfrak{f}} \mathbb{R}, D, D^{*}, \tilde{g}\right)$ be a statistical warped product manifold, where $\left(\mathbb{R}, \nabla^{\mathbb{R}}, d z^{2}\right)$ is a trivial statistical manifold of dimension one and $\operatorname{dim}\left(N_{1}\right)=k$. For $X, Y \in \chi\left(N_{1}\right)$, we have:

(a) $\tilde{\operatorname{Ric}}(X, Y)=\operatorname{Ric}_{1}(X, Y)-\mathfrak{f}^{-1} \operatorname{Hess}_{\mathfrak{f}}(X, Y)$,

(b) $\tilde{R} i c(X, \partial z)=0$,

(c) $\tilde{R} i c(\partial z, \partial z)=-\mathfrak{f}(\Delta \mathfrak{f}) g_{2}(\partial z, \partial z)$.

Theorem 3. Let $\zeta=\left(\zeta_{1}, \partial z\right) \in \chi(\tilde{N})$ be a vector field on statistical warped product manifold $\left(\tilde{N}=N_{1} \times_{\mathfrak{f}} \mathbb{R}, D, D^{*}, \tilde{g}=g_{1}+\mathfrak{f}^{2} d z^{2}\right)$ with $\operatorname{dim}(\mathbb{R})=1$ and $\operatorname{dim}\left(N_{1}\right)=k$. Suppose that Hess $\mathfrak{f}=0$. Then, $(\zeta, \lambda)$ is a statistical soliton on $\tilde{N}$ if and only if $\left(\zeta_{1}, \lambda=\mathfrak{f}^{-1}(\Delta \mathfrak{f})\right)$ is a statistical soliton on $N_{1}$.

Proof. Since $g_{2}\left(\nabla_{\partial z}^{N_{1}} \partial z, \partial z\right)=0$ and $\operatorname{Ric}_{2}(\partial z, \partial z)=0$, then by using Equation (15) and Lemma 4, we get:

$$
\nabla^{N_{1}} \zeta_{1}+R i c_{1}-\mathfrak{f}(\Delta \mathfrak{f}) g_{2}+\lambda g_{1}+\lambda \mathfrak{f}^{2} g_{2}=0
$$

Therefore, we have:

$$
\nabla^{N_{1}} \zeta_{1}+R i c_{1}+\lambda g_{1}=0
$$

Furthermore, $\mathfrak{f}^{-1}(\Delta \mathfrak{f})=\lambda=$ constant. Putting this into (19), we get:

$$
\nabla^{N_{1}} \zeta_{1}+\operatorname{Ric}_{1}+\mathfrak{f}^{-1}(\Delta \mathfrak{f}) g_{1}=0
$$

Similarly, by using (16), we obtain:

$$
\nabla^{N_{1} *} \zeta_{1}+R i c_{1}^{*}+\mathfrak{f}^{-1}\left(\Delta^{*} \mathfrak{f}\right) g_{1}=0
$$

Since $\mathfrak{f}^{-1}(\Delta \mathfrak{f})$ is constant, $\left(N_{1}, g_{1}, \zeta_{1}, \lambda=\mathfrak{f}^{-1}(\Delta \mathfrak{f})\right)$ is a statistical soliton.

Conversely, if $\left(\zeta_{1}, \lambda=\mathfrak{f}^{-1}(\Delta \mathfrak{f})\right)$ is a statistical soliton on $N_{1}$, then:

$$
\begin{aligned}
\nabla^{N_{1}} \zeta_{1} & +\nabla^{N_{2}} \partial z+\text { Ric }_{1}-\mathfrak{f}^{-1} k_{2} \text {Hess }_{\mathfrak{f}}+\text { Ric }_{2} \\
& -\left[\mathfrak{f}(\Delta \mathfrak{f})+\left(k_{2}-1\right) \| \text { grad } \mathfrak{f} \|^{2}\right] g_{2} \\
= & \nabla^{N_{1}} \zeta_{1}+R i c_{1}+\mathfrak{f}^{-1}(\Delta \mathfrak{f}) g_{1}-\mathfrak{f}^{-1}(\Delta \mathfrak{f}) g_{1}-\mathfrak{f}(\Delta \mathfrak{f}) g_{2} \\
= & -\mathfrak{f}^{-1}(\Delta \mathfrak{f}) g_{1}-\mathfrak{f}(\Delta \mathfrak{f}) g_{2}=-\mathfrak{f}^{-1}(\Delta \mathfrak{f})\left(g_{1}+g_{2}\right) \\
= & -\lambda \tilde{g} .
\end{aligned}
$$
on $\tilde{N}$.

Thus, $D \zeta+\tilde{\mathcal{Q}}+\lambda I=0$. Similarly, $D^{*} \zeta+\tilde{\mathcal{Q}}^{*}+\lambda I=0$. Hence, $(\zeta, \lambda)$ is a statistical soliton

An immediate consequence of Theorem 3 is as follows:

Corollary 1. Let $(\tilde{N}, \tilde{g}, \zeta, \lambda)$ be a Statistical soliton on statistical manifold $\left(\tilde{N}=N_{1} \times_{\mathfrak{f}} \mathbb{R}, D, D^{*}, \tilde{g}=g_{1}+\right.$ $\left.\mathfrak{f}^{2} d z^{2}\right)$ with $\operatorname{dim}(\mathbb{R})=1$ and $\operatorname{dim}\left(N_{1}\right)=k$. If $\operatorname{Hess}_{\mathfrak{f}}=\varrho g_{1}, \varrho \in C^{\infty}\left(N_{1}\right)$, then $\left(N_{1}, g_{1}, \zeta_{1}, \mathfrak{f}^{-1}(\Delta \mathfrak{f})-\mathfrak{f}^{-1} \varrho\right)$ is a statistical soliton.

\section{B.Y. Chen Inequality}

A universal sharp inequality for submanifolds in a Riemannian manifold of constant sectional curvature was established in [38], known as the first Chen inequality. The main purpose of this section 
is to establish the corresponding inequality for statistical warped product manifolds statistically immersed in a statistical manifold of constant curvature.

Let $\varphi: N=N_{1} \times_{\mathfrak{f}} N_{2} \rightarrow \tilde{N}(\tilde{c})$ be an isometric statistical immersion of a warped product $N_{1} \times_{f} N_{2}$ into a statistical manifold of constant sectional curvature $\tilde{c}$. We denote by $r, k$, and $m=r+k$ the dimensions of $N_{1}, N_{2}$, and $N_{1} \times N_{2}$, respectively. Since $N_{1} \times{ }_{\mathfrak{f}} N_{2}$ is a statistical warped product, we have:

$$
\nabla_{E_{1}} E_{2}=\nabla_{E_{2}} E_{1}=\left(E_{1} \ln \mathfrak{f}\right) E_{2}
$$

for unit vector fields $E_{1}$ and $E_{2}$ tangent to $N_{1}$ and $N_{2}$, respectively. Hence, we derive:

$$
\mathbb{K}\left(E_{1} \wedge E_{2}\right)=\frac{1}{\mathfrak{f}}\left\{\left(\nabla_{E_{1}} E_{1}\right) \mathfrak{f}-E_{1}^{2} \mathfrak{f}\right\} .
$$

If we choose a local orthonormal frame $\left\{e_{1}, \ldots, e_{m}\right\}$ such that $\left\{e_{1}, \ldots, e_{r}\right\}$ are tangent to $N_{1}$ and $\left\{e_{r+1}, \ldots, e_{r+k}=e_{m}\right\}$ are tangent to $N_{2}$, then we have:

$$
\frac{\Delta \mathfrak{f}}{\mathfrak{f}}=\sum_{i=1}^{r} \mathbb{K}\left(e_{i} \wedge e_{j}\right),
$$

for each $j=r+1, \ldots, m$.

On the other hand, let $E_{1}$ and $E_{2}$ be two unit local vector fields tangent to $N_{1}$ and $N_{2}$, respectively, such that $e_{1}=E_{1}$ and $e_{r+1}=E_{2}$. By taking into account Equations (3), (6), and (9), we derive (7) as follows:

$$
\begin{aligned}
\mathbb{K}^{\nabla, \nabla^{*}}\left(e_{1} \wedge\right. & \left.e_{r+1}\right)=\frac{\tilde{c}}{2}\left\{2 g\left(e_{r+1}, e_{r+1}\right) g\left(e_{1}, e_{1}\right)-2 g\left(e_{1}, e_{r+1}\right) g\left(e_{r+1}, e_{1}\right)\right\} \\
+ & \frac{1}{2}\left\{g\left(h^{*}\left(e_{1}, e_{1}\right), h\left(e_{r+1}, e_{r+1}\right)\right)\right. \\
& \left.+g\left(h\left(e_{1}, e_{1}\right), h^{*}\left(e_{r+1}, e_{r+1}\right)\right)-2 g\left(h\left(e_{1}, e_{r+1}\right), h^{*}\left(e_{1}, e_{r+1}\right)\right)\right\} \\
= & \tilde{c}+\frac{1}{2} \sum_{a=m+1}^{n}\left\{h_{11}^{* a} h_{r+1, r+1}^{a}+h_{11}^{a} h_{r+1, r+1}^{* a}-2 h_{1, r+1}^{a} h_{1, r+1}^{* a}\right\} .
\end{aligned}
$$

We rewrite the terms of the RHS of the previous equation as:

$$
\begin{aligned}
\mathbb{K}^{\nabla, \nabla^{*}}\left(e_{1} \wedge e_{r+1}\right)= & \tilde{c}+\frac{1}{2} \sum_{a=m+1}^{n}\left\{\left(h_{11}^{a}+h_{11}^{* a}\right)\left(h_{r+1, r+1}^{a}+h_{r+1, r+1}^{* a}\right)\right. \\
& -\left(h_{1, r+1}^{a}+h_{1, r+1}^{* a}\right)^{2}+\left(h_{1, r+1}^{a}\right)^{2}+\left(h_{1, r+1}^{* a}\right)^{2} \\
& \left.-h_{11}^{a} h_{r+1, r+1}^{a}-h_{11}^{* a} h_{r+1, r+1}^{* a}\right\} .
\end{aligned}
$$

Since, $2 h^{0}=h+h^{*}$, we get:

$$
\begin{aligned}
\mathbb{K}^{\nabla, \nabla^{*}}\left(e_{1} \wedge e_{r+1}\right)= & \tilde{c}+\frac{1}{2} \sum_{a=m+1}^{n}\left\{4 h_{11}^{0 a} h_{r+1, r+1}^{0 a}\right. \\
& -\left(h_{11}^{a} h_{r+1, r+1}^{a}-\left(h_{1, r+1}^{a}\right)^{2}\right) \\
& \left.-\left(h_{11}^{* a} h_{r+1, r+1}^{* a}-\left(h_{1, r+1}^{* a}\right)^{2}\right)-4\left(h_{1, r+1}^{0 a}\right)^{2}\right\} .
\end{aligned}
$$


Thus, we have:

$$
\begin{aligned}
& \mathbb{K}^{\nabla, \nabla^{*}}\left(e_{1} \wedge e_{r+1}\right)=\tilde{c}+\sum_{a=m+1}^{n}\left\{2\left(h_{11}^{0 a} h_{r+1, r+1}^{0 a}-\left(h_{1, r+1}^{0 a}\right)^{2}\right)\right. \\
& \left.-\frac{1}{2}\left(h_{11}^{a} h_{r+1, r+1}^{a}-\left(h_{1, r+1}^{a}\right)^{2}\right)-\frac{1}{2}\left(h_{11}^{* a} h_{r+1, r+1}^{* a}-\left(h_{1, r+1}^{* a}\right)^{2}\right)\right\} .
\end{aligned}
$$

Using the Gauss equation for the Levi-Civita connection, we arrive at:

$$
\mathbb{K}^{0}\left(e_{1} \wedge e_{r+1}\right)=\tilde{c}-\sum_{a=m+1}^{n}\left\{\left(h_{1, r+1}^{0 a}\right)^{2}-h_{11}^{0 a} h_{r+1, r+1}^{0 a}\right\},
$$

which can be rewritten as:

$$
\sum_{a=m+1}^{n}\left\{\left(h_{1, r+1}^{0 a}\right)^{2}-h_{11}^{0 a} h_{r+1, r+1}^{0 a}\right\}=\mathbb{K}^{0}\left(e_{1} \wedge e_{r+1}\right)-\tilde{c} .
$$

Substituting (23) into (22), we get:

$$
\begin{aligned}
\mathbb{K}^{\nabla, \nabla^{*}}\left(e_{1} \wedge e_{r+1}\right)= & 2 \mathbb{K}^{0}\left(e_{1} \wedge e_{r+1}\right)-\tilde{c}-\frac{1}{2} \sum_{a=m+1}^{n}\left\{h_{11}^{a} h_{r+1, r+1}^{a}\right. \\
& \left.-\left(h_{1, r+1}^{a}\right)^{2}+h_{11}^{* a} h_{r+1, r+1}^{* a}-\left(h_{1, r+1}^{* a}\right)^{2}\right\} .
\end{aligned}
$$

Furthermore, we derive (8) as:

$$
\begin{aligned}
\sigma^{\nabla, \nabla^{*}}= & \frac{m(m-1) \tilde{c}}{2}+\frac{1}{2} \sum_{a=m+1}^{n} \sum_{i<j}\left\{h_{i i}^{* a} h_{j j}^{a}+h_{i i}^{a} h_{j j}^{* a}-2 h_{i j}^{a} h_{i j}^{* a}\right\} \\
= & \frac{m(m-1) \tilde{c}}{2}+\frac{1}{2} \sum_{a=m+1}^{n} \sum_{i<j}\left\{\left(h_{i i}^{a}+h_{i i}^{* a}\right)\left(h_{j j}^{a}+h_{j j}^{* a}\right)\right. \\
& \left.-h_{i i}^{a} h_{j j}^{a}-h_{i i}^{* a} h_{j j}^{* a}-\left(h_{i j}^{a}+h_{i j}^{* a}\right)^{2}+\left(h_{i j}^{a}\right)^{2}+\left(h_{i j}^{* a}\right)^{2}\right\} .
\end{aligned}
$$

By a similar argument as above, we deduce that:

$$
\begin{array}{r}
\sigma^{\nabla, \nabla^{*}}=\frac{m(m-1) \tilde{c}}{2}+\frac{1}{2} \sum_{a=m+1}^{n} \sum_{i<j}\left\{2\left(h_{i i}^{0 a} h_{j j}^{0 a}-\left(h_{i j}^{0 a}\right)^{2}\right)\right. \\
\left.-\frac{1}{2}\left(h_{i i}^{a} h_{j j}^{a}-\left(h_{i j}^{a}\right)^{2}\right)-\frac{1}{2}\left(h_{i i}^{* a} h_{j j}^{* a}-\left(h_{i j}^{* a}\right)^{2}\right)\right\} .
\end{array}
$$

Again by the Gauss equation for the Levi-Civita connection, we find that:

$$
\sigma^{0}=\frac{m(m-1) \tilde{c}}{2}+\sum_{a=m+1}^{n} \sum_{i<j}\left\{h_{i i}^{0 a} h_{j j}^{0 a}-\left(h_{i j}^{0 a}\right)^{2}\right\},
$$

or:

$$
\sum_{a=m+1}^{n} \sum_{i<j}\left\{h_{i i}^{0 a} h_{j j}^{0 a}-\left(h_{i j}^{0 a}\right)^{2}\right\}=\sigma^{0}-\frac{m(m-1) \tilde{c}}{2} .
$$


Inserting (26) into (25), we have:

$$
\begin{aligned}
\sigma^{\nabla, \nabla^{*}}= & 2 \sigma^{0}-\frac{m(m-1) \tilde{c}}{2}-\frac{1}{2} \sum_{a=m+1}^{n} \sum_{i<j}\left\{h_{i i}^{a} h_{j j}^{a}-\left(h_{i j}^{a}\right)^{2}\right. \\
& \left.+h_{i i}^{* a} h_{j j}^{* a}-\left(h_{i j}^{* a}\right)^{2}\right\} .
\end{aligned}
$$

By subtracting (24) from (27), we can state the following result:

Lemma 5. Let $N=N_{1} \times_{\mathfrak{f}} N_{2}$ be an m-dimensional statistical warped product submanifold immersed into an $n$-dimensional statistical manifold of constant sectional curvature $\tilde{c}$. Then:

$$
\begin{aligned}
\sigma^{\nabla, \nabla^{*}}-\mathbb{K}^{\nabla, \nabla^{*}}\left(e_{1} \wedge e_{r+1}\right)= & 2\left(\sigma^{0}-\mathbb{K}^{0}\left(e_{1} \wedge e_{r+1}\right)\right)-\frac{(m-2)(m+1) \tilde{c}}{2} \\
& -\frac{1}{2} \sum_{a=m+1}^{n} \sum_{i<j}\left\{h_{i i}^{a} h_{j j}^{a}-\left(h_{i j}^{a}\right)^{2}+h_{i i}^{* a} h_{j j}^{* a}\right. \\
& \left.-\left(h_{i j}^{* a}\right)^{2}\right\}+\frac{1}{2} \sum_{a=m+1}^{n}\left\{h_{11}^{a} h_{r+1, r+1}^{a}-\left(h_{1, r+1}^{a}\right)^{2}\right. \\
& \left.+h_{11}^{* a} h_{r+1, r+1}^{* a}-\left(h_{1, r+1}^{* a}\right)^{2}\right\} .
\end{aligned}
$$

Further, we have:

$$
\begin{aligned}
\sigma^{\nabla, \nabla^{*}}-\mathbb{K}^{\nabla, \nabla^{*}}\left(e_{1} \wedge e_{r+1}\right) \geq & 2\left(\sigma^{0}-\mathbb{K}^{0}\left(e_{1} \wedge e_{r+1}\right)\right)-\frac{(m-2)(m+1) \tilde{c}}{2} \\
& -\frac{1}{2} \sum_{a=m+1}^{n} \sum_{i<j}\left\{h_{i i}^{a} h_{j j}^{a}+h_{i i}^{* a} h_{j j}^{* a}\right\} \\
& +\frac{1}{2} \sum_{a=m+1}^{n}\left\{h_{11}^{a} h_{r+1, r+1}^{a}+h_{11}^{* a} h_{r+1, r+1}^{* a}\right\}
\end{aligned}
$$

or we write it as:

$$
\begin{aligned}
2\left(\sigma^{0}-\mathbb{K}^{0}\left(e_{1} \wedge e_{r+1}\right)\right) \leq & \sigma^{\nabla, \nabla^{*}}-\mathbb{K}^{\nabla, \nabla^{*}}\left(e_{1} \wedge e_{r+1}\right)+\frac{(m-2)(m+1) \tilde{c}}{2} \\
& +\frac{1}{2} \sum_{a=m+1}^{n}\left\{\sum_{i<j}\left\{h_{i i}^{a} h_{j j}^{a}\right\}-h_{11}^{a} h_{r+1, r+1}^{a}\right\} \\
& +\frac{1}{2} \sum_{a=m+1}^{n}\left\{\sum_{i<j}\left\{h_{i i}^{* a} h_{j j}^{* a}\right\}-h_{11}^{* a} h_{r+1, r+1}^{* a}\right\} .
\end{aligned}
$$

We use an optimization technique: For $a \in[m+1, n]$, we consider the quadratic forms:

$$
\phi_{a}: \mathbb{R}^{m} \rightarrow \mathbb{R}, \phi_{a}^{*}: \mathbb{R}^{m} \rightarrow \mathbb{R}
$$

given by:

$$
\phi_{a}\left(h_{11}^{a}, \ldots, h_{m m}^{a}\right)=\sum_{i<j}\left\{h_{i i}^{a} h_{j j}^{a}\right\}-h_{11}^{a} h_{r+1, r+1}^{a}
$$

and:

$$
\phi_{a}^{*}\left(h_{11}^{* a}, \ldots, h_{m m}^{* a}\right)=\sum_{i<j}\left\{h_{i i}^{* a} h_{j j}^{* a}\right\}-h_{11}^{* a} h_{r+1, r+1}^{* a}
$$


The constrained extremum problem is $\max \phi_{a}$ subject to:

$$
Q: h_{11}^{a}+\cdots+h_{m m}^{a}=t^{a}, \quad\left(t^{a} \text { is any constant }\right)
$$

The partial derivatives of $\phi_{a}$ are:

$$
\begin{aligned}
& \frac{\partial \phi_{a}}{\partial h_{11}^{a}}=\sum_{i=2}^{m} h_{i i}^{a}-h_{r+1, r+1}^{a}, \\
& \frac{\partial \phi_{a}}{\partial h_{r+1, r+1}^{a}}=\sum_{i \in \overline{1, m} r+1} h_{i i}^{a}-h_{11}^{a} \text {, } \\
& \frac{\partial \phi_{a}}{\partial h_{l l}^{a}}=\sum_{i \in \frac{1, m}{\{l\}}} h_{i i}^{a}, \quad l \in[r+2, m] .
\end{aligned}
$$

For an optimal solution $\left(h_{11}^{a}, \ldots, h_{m m}^{a}\right)$ of the above problem and grad $\left(\phi_{a}\right)$ normal at $Q$, we obtain:

$$
\left(h_{11}^{a}, h_{22}^{a}, \ldots, h_{m m}^{a}\right)=\left(0, \alpha^{a}, \ldots, \alpha^{a}\right)
$$

As $t^{a}=\sum_{i=1}^{m} h_{i i}^{a}=(m-1) \alpha^{a}$, then we have:

$$
\alpha^{a}=\frac{t^{a}}{m-1} .
$$

As $\phi_{a}$ is obtained from the similar function studied in [39] by subtracting some square terms, $\phi_{a} \mid Q$ will have the Hessian semi-negative definite. Consequently, the point in (31), together with (32) is a global maximum point, and hence, we calculate:

$$
\begin{aligned}
\phi_{a} & \leq \frac{(m-1)(m-2)\left(\alpha^{a}\right)^{2}}{2} \\
& =\frac{(m-2)\left(t^{a}\right)^{2}}{2(m-1)}=\frac{m^{2}(m-2)}{2(m-1)}\left(\mathcal{H}^{a}\right)^{2} .
\end{aligned}
$$

Similarly, one gets:

$$
\phi_{a}^{*} \leq \frac{m^{2}(m-2)}{2(m-1)}\left(\mathcal{H}^{* a}\right)^{2},
$$

by considering (30) and the constrained extremum problem max $\phi_{a}^{*}$ subject to:

$$
Q^{*}: h_{11}^{* a}+\cdots+h_{m m}^{* a}=t^{* a}, \quad\left(t^{* a} \text { is any constant }\right) \text {. }
$$

Thus, (28) becomes:

$$
\begin{aligned}
2\left(\sigma^{0}-\mathbb{K}^{0}\left(e_{1} \wedge e_{r+1}\right)\right) \leq & \sigma^{\nabla, \nabla^{*}}-\mathbb{K}^{\nabla, \nabla^{*}}\left(e_{1} \wedge e_{r+1}\right)+\frac{(m-2)(m+1) \tilde{c}}{2} \\
& +\frac{m^{2}(m-2)}{4(m-1)}\left(\|\mathcal{H}\|^{2}+\left\|\mathcal{H}^{*}\right\|^{2}\right)
\end{aligned}
$$

By summarizing, we state the following: 
Theorem 4. Let $N=N_{1} \times_{\mathfrak{f}} N_{2}$ be an m-dimensional statistical warped product submanifold immersed into an n-dimensional statistical manifold of constant sectional curvature $\tilde{c}$. Then:

$$
\begin{aligned}
\sigma^{\nabla, \nabla^{*}}-\mathbb{K}^{\nabla, \nabla^{*}}\left(e_{1} \wedge e_{r+1}\right) \geq & 2\left(\sigma^{0}-\mathbb{K}^{0}\left(e_{1} \wedge e_{r+1}\right)\right)-\frac{(m-2)(m+1) \tilde{c}}{2} \\
& -\frac{m^{2}(m-2)}{4(m-1)}\left(\|\mathcal{H}\|^{2}+\left\|\mathcal{H}^{*}\right\|^{2}\right) .
\end{aligned}
$$

By using (20), we obtain:

$$
\begin{aligned}
\mathbb{K}^{\nabla, \nabla^{*}}\left(e_{1} \wedge e_{r+1}\right) & =\frac{1}{2}\left(\mathbb{K}\left(e_{1} \wedge e_{r+1}\right)+\mathbb{K}^{*}\left(e_{1} \wedge e_{r+1}\right)\right) \\
& =\frac{1}{2 \mathfrak{f}}\left\{\left(\nabla_{e_{1}} e_{1}\right) \mathfrak{f}-e_{1}^{2} \mathfrak{f}+\left(\nabla_{e_{1}}^{*} e_{1}\right) \mathfrak{f}-e_{1}^{2} \mathfrak{f}\right\} .
\end{aligned}
$$

For $b=1,2, \ldots, r$, we also have:

$$
\mathbb{K}^{\nabla, \nabla^{*}}\left(e_{b} \wedge e_{r+1}\right)=\frac{1}{2 \mathfrak{f}}\left\{\left(\nabla_{e_{b}} e_{b}\right) \mathfrak{f}-e_{b}^{2} \mathfrak{f}+\left(\nabla_{e_{b}}^{*} e_{b}\right) \mathfrak{f}-e_{b}^{2} \mathfrak{f}\right\} .
$$

By summing up $b$ from one to $r$, we find that:

$$
\sum_{b=1}^{r} \frac{1}{2 \mathfrak{f}}\left\{\left(\nabla_{e_{b}} e_{b}\right) \mathfrak{f}-e_{b}^{2} \mathfrak{f}+\left(\nabla_{e_{b}}^{*} e_{b}\right) \mathfrak{f}-e_{b}^{2} \mathfrak{f}\right\}=\frac{1}{2}\left(\frac{\Delta^{N_{1}} \mathfrak{f}}{\mathfrak{f}}+\frac{\Delta^{N_{1} *} \mathfrak{f}}{\mathfrak{f}}\right)=\frac{\Delta^{N_{1} 0} \mathfrak{f}}{\mathfrak{f}}
$$

where $\Delta^{N_{1}}$ and $\Delta^{N_{1} *}$ are dual Laplacians of $N_{1}$ and $\Delta^{N_{1} 0}$ denotes the Laplacian operator of $N_{1}$ for the Levi-Civita connection [37]. Thus, we have:

Theorem 5. Let $N=N_{1} \times_{\mathfrak{f}} N_{2}$ be an m-dimensional statistical warped product submanifold immersed into an $n$-dimensional statistical manifold of constant sectional curvature $\tilde{c}$. Then, the scalar curvature $\sigma^{\nabla, \nabla^{*}}$ of $N$ satisfies:

$$
\begin{aligned}
\sigma^{\nabla, \nabla^{*}} \geq & 2 \sigma^{0}-\frac{\Delta^{N_{1} 0} \mathfrak{f}}{r \mathfrak{f}}-\frac{(m-2)(m+1) \tilde{c}}{2} \\
& -\frac{m^{2}(m-2)}{4(m-1)}\left(\|\mathcal{H}\|^{2}+\left\|\mathcal{H}^{*}\right\|^{2}\right) .
\end{aligned}
$$

\section{Optimal Casorati Inequality}

Let $\left\{e_{1}, \ldots, e_{m}\right\}$ and $\left\{e_{m+1}, \ldots, e_{n}\right\}$ be respectively the orthonormal basis of $T_{p} N$ and $T_{p}^{\perp} N, p \in N$. Then, the squared norm of second fundamental forms $h$ and $h^{*}$ is denoted by $\mathcal{C}$ and $\mathcal{C}^{*}$, respectively, called the Casorati curvatures of $N$ in $\tilde{N}$. Therefore, we have:

$$
\mathcal{C}=\frac{1}{m}\|h\|^{2}, \quad \mathcal{C}^{*}=\frac{1}{m}\left\|h^{*}\right\|^{2},
$$

where:

$$
\|h\|^{2}=\sum_{a=m+1}^{n} \sum_{i, j=1}^{m}\left(h_{i j}^{a}\right)^{2},\left\|h^{*}\right\|^{2}=\sum_{a=m+1}^{n} \sum_{i, j=1}^{m}\left(h_{i j}^{* a}\right)^{2} .
$$

If $W$ is a $q$-dimensional subspace of $T N, q \geq 2$, and $\left\{e_{1}, \ldots, e_{q}\right\}$ an orthonormal basis of $W$. Then, the scalar curvature of the $q$-plane section $W$ is:

$$
\sigma^{\nabla, \nabla^{*}}(W)=\sum_{1 \leq i<j \leq q} S\left(e_{i}, e_{j}, e_{j}, e_{i}\right)
$$


and the Casorati curvatures of the subspace $W$ are as follows:

$$
\mathcal{C}(W)=\frac{1}{q} \sum_{a=m+1}^{n} \sum_{i, j=1}^{q}\left(h_{i j}^{a}\right)^{2}, \mathcal{C}^{*}(W)=\frac{1}{q} \sum_{a=m+1}^{n} \sum_{i, j=1}^{q}\left(h_{i j}^{* a}\right)^{2}
$$

(1) The normalized Casorati curvatures $\delta_{\mathcal{C}}(m-1)$ and $\delta_{\mathcal{C}}^{*}(m-1)$ are defined as:

$$
\begin{aligned}
{\left[\delta_{\mathcal{C}}(m-1)\right]_{p} } & =\frac{1}{2} \mathcal{C}_{p}+\left(\frac{m+1}{2 m}\right) \inf \left\{\mathcal{C}(W) \mid W: \text { a hyperplane of } T_{p} N\right\}, \\
\text { and }\left[\delta_{\mathcal{C}}^{*}(m-1)\right]_{p} & =\frac{1}{2} \mathcal{C}_{p}^{*}+\left(\frac{m+1}{2 m}\right) \inf \left\{\mathcal{C}^{*}(W) \mid W: \text { a hyperplane of } T_{p} N\right\} .
\end{aligned}
$$

(2) The normalized Casorati curvatures $\widehat{\delta}_{\mathcal{C}}(m-1)$ and $\widehat{\delta}_{\mathcal{C}}^{*}(m-1)$ are defined as:

$$
\begin{array}{r}
{\left[\widehat{\delta}_{\mathcal{C}}(m-1)\right]_{p}=2 \mathcal{C}_{p}-\left(\frac{2 m-1}{2 m}\right) \sup \left\{\mathcal{C}(W) \mid W: \text { a hyperplane of } T_{p} N\right\}} \\
\text { and }\left[\widehat{\delta}_{\mathcal{C}}^{*}(m-1)\right]_{p}=2 \mathcal{C}_{p}^{*}-\left(\frac{2 m-1}{2 m}\right) \sup \left\{\mathcal{C}^{*}(W) \mid W: \text { a hyperplane of } T_{p} N\right\}
\end{array}
$$

Let $\varphi: N=N_{1} \times_{\mathfrak{f}} N_{2} \rightarrow \tilde{N}(\tilde{c})$ be an isometric statistical immersion of a warped product $N_{1} \times_{\mathfrak{f}} N_{2}$ into a statistical manifold of constant sectional curvature $\tilde{c}$. If we chose a local orthonormal frame $\left\{e_{1}, \ldots, e_{m}\right\}$ such that $\left\{e_{1}, \ldots, e_{r}\right\}$ are tangent to $N_{1}$ and $\left\{e_{r+1}, \ldots, e_{r+k}=e_{m}\right\}$ are tangent to $N_{2}$, then the two partial mean curvature vectors $\mathcal{H}_{1}$ (resp. $\mathcal{H}_{1}^{*}$ ) and $\mathcal{H}_{2}$ (resp. $\mathcal{H}_{2}^{*}$ ) of $N$ are given by:

$$
\mathcal{H}_{1}=\frac{1}{r} \sum_{i=1}^{r} h\left(e_{i}, e_{i}\right), \quad \mathcal{H}_{1}^{*}=\frac{1}{r} \sum_{i=1}^{r} h^{*}\left(e_{i}, e_{i}\right),
$$

and:

$$
\mathcal{H}_{2}=\frac{1}{k} \sum_{j=1}^{k} h\left(e_{r+j}, e_{r+j}\right), \quad \mathcal{H}_{2}^{*}=\frac{1}{k} \sum_{j=1}^{k} h^{*}\left(e_{r+j}, e_{r+j}\right) .
$$

Furthermore, the Casorati curvatures are:

$$
\mathcal{C}_{1}=\frac{1}{r} \sum_{a=m+1}^{n} \sum_{i, j=1}^{r}\left(h_{i j}^{a}\right)^{2}, \quad \mathcal{C}_{1}^{*}=\frac{1}{r} \sum_{a=m+1}^{n} \sum_{i, j=1}^{r}\left(h_{i j}^{* a}\right)^{2},
$$

and:

$$
\mathcal{C}_{2}=\frac{1}{k} \sum_{a=m+1}^{n} \sum_{i, j=1}^{k}\left(h_{r+i r+j}^{a}\right)^{2}, \quad \mathcal{C}_{2}^{*}=\frac{1}{k} \sum_{a=m+1}^{n} \sum_{i, j=1}^{k}\left(h_{r+i r+j}^{* a}\right)^{2}
$$

Equation (21) implies:

$$
\frac{k \Delta^{N_{1} 0_{\mathfrak{f}}}}{\mathfrak{f}}=\sigma^{\nabla, \nabla^{*}}-\sum_{1 \leq i \leq j \leq r} \mathbb{K}^{\nabla, \nabla^{*}}\left(e_{i} \wedge e_{j}\right)-\sum_{r+1 \leq l \leq s \leq m} \mathbb{K}^{\nabla, \nabla^{*}}\left(e_{l} \wedge e_{s}\right) .
$$


By using (8), the previous equation becomes:

$$
\begin{aligned}
2 \sigma^{\nabla, \nabla^{*}}= & \frac{k \Delta^{N_{1} 0} \mathfrak{f}}{\mathfrak{f}}+r(r-1) \tilde{c}+k(k-1) \tilde{c}+2 r^{2}\left\|\mathcal{H}_{1}^{0}\right\|^{2} \\
& -\frac{r^{2}}{2}\left(\left\|\mathcal{H}_{1}\right\|^{2}+\left\|\mathcal{H}_{1}^{*}\right\|^{2}\right)-\frac{k^{2}}{2}\left(\left\|\mathcal{H}_{2}\right\|^{2}+\left\|\mathcal{H}_{2}^{*}\right\|^{2}\right) \\
& +2 k^{2}\left\|\mathcal{H}_{2}^{0}\right\|^{2}-2 r \mathcal{C}_{1}^{0}+\frac{r}{2}\left(\mathcal{C}_{1}+\mathcal{C}_{1}^{*}\right) \\
& -2 k \mathcal{C}_{2}^{0}+\frac{k}{2}\left(\mathcal{C}_{2}+\mathcal{C}_{2}^{*}\right) .
\end{aligned}
$$

We define a polynomial $P$ in terms of the components of the second fundamental form $h^{0}$ (with respect to the Levi-Civita connection) of $N$.

$$
\begin{aligned}
P= & 2 r(r-1) \mathcal{C}_{1}^{0}+\left(r^{2}-1\right) \mathcal{C}_{1}^{0}\left(W_{1}\right)+\frac{r}{2}\left(\mathcal{C}_{1}+\mathcal{C}_{1}^{*}\right) \\
& +2 k(k-1) \mathcal{C}_{2}^{0}+\left(k^{2}-1\right) \mathcal{C}_{2}^{0}\left(W_{2}\right)+\frac{k}{2}\left(\mathcal{C}_{2}+\mathcal{C}_{2}^{*}\right) \\
& +\frac{k \Delta^{N_{1} 0} \mathfrak{f}}{\mathfrak{f}}+r(r-1) \tilde{c}+k(k-1) \tilde{c}-\frac{r^{2}}{2}\left(\left\|\mathcal{H}_{1}\right\|^{2}+\left\|\mathcal{H}_{1}^{*}\right\|^{2}\right) \\
& -\frac{k^{2}}{2}\left(\left\|\mathcal{H}_{2}\right\|^{2}+\left\|\mathcal{H}_{2}^{*}\right\|^{2}\right)-2 \sigma^{\nabla, \nabla^{*}}
\end{aligned}
$$

Without loss of generality, we assume that $W_{1}$ and $W_{2}$ are respectively spanned by $\left\{e_{1}, \ldots, e_{r-1}\right\}$ and $\left\{e_{r+1}, \ldots, e_{r+k-1}\right\}$. Then, by (36) and (37), we derive:

$$
\begin{aligned}
P= & \sum_{a=m+1}^{n}\left\{\sum_{i, j=1}^{r} \frac{r+3}{2}\left(h_{i j}^{0 a}\right)^{2}+\frac{r+1}{2} \sum_{i, j=1}^{r-1}\left(h_{i j}^{0 a}\right)^{2}-2\left(\sum_{i=1} h_{i i}^{0 a}\right)^{2}\right\} \\
& +\sum_{a=m+1}^{n}\left\{\sum_{l, s=1}^{k} \frac{k+3}{2}\left(h_{l s}^{0 a}\right)^{2}+\frac{k+1}{2} \sum_{l, s=1}^{k-1}\left(h_{l s}^{0 a}\right)^{2}-2\left(\sum_{l=1} h_{l l}^{0 a}\right)^{2}\right\} \\
= & \sum_{a=m+1}^{n}\left\{2(r+2) \sum_{1 \leq i<j \leq r-1}\left(h_{i j}^{0 a}\right)^{2}+(r+3) \sum_{i=1}^{r-1}\left(h_{i r}^{0 a}\right)^{2}\right. \\
& \left.+r \sum_{i=1}^{r-1}\left(h_{i i}^{0 a}\right)^{2}-4 \sum_{1 \leq i<j \leq r}\left(h_{i i}^{0 a} h_{j j}^{0 a}\right)+\frac{r-1}{2}\left(h_{r r}^{0 a}\right)^{2}\right\} \\
& +\sum_{a=m+1}^{n}\left\{2(k+2) \sum_{1 \leq l<s \leq k-1}\left(h_{l s}^{0 a}\right)^{2}+(k+3) \sum_{l=1}^{k-1}\left(h_{l k}^{0 a}\right)^{2}\right. \\
& \left.+k \sum_{l=1}^{k-1}\left(h_{l l}^{0 a}\right)^{2}-4 \sum_{1 \leq l<s \leq k}\left(h_{l l}^{0 a} h_{s s}^{0 a}\right)+\frac{k-1}{2}\left(h_{k k}^{0 a}\right)^{2}\right\} \\
\geq & \sum_{a=m+1}^{n}\left\{\sum_{i=1}^{r-1} r\left(h_{i i}^{0 a}\right)^{2}+\frac{r-1}{2}\left(h_{r r}^{0 a}\right)^{2}-4 \sum_{1 \leq i<j \leq r} h_{i i}^{0 a} h_{j j}^{0 a}\right\} \\
& +\sum_{a=m+1}^{n}\left\{\sum_{l=1}^{k-1} k\left(h_{l l}^{0 a}\right)^{2}+\frac{k-1}{2}\left(h_{k k}^{0 a}\right)^{2}-4 \sum_{1 \leq l<s \leq k} h_{l l}^{0 a} h_{s s}^{0 a}\right\} .
\end{aligned}
$$


For any $a \in\{m+1, \ldots, n\}$, we define two quadratic forms $\phi_{a}: \mathbb{R}^{r} \rightarrow \mathbb{R}$ and $\varphi_{a}: \mathbb{R}^{k} \rightarrow \mathbb{R}$ by:

$$
\begin{aligned}
& \phi_{a}\left(h_{11}^{0 a}, h_{22}^{0 a}, \ldots, h_{r-1, r-1}^{0 a}, h_{r r}^{0 a}\right) \\
& \quad=\sum_{i=1}^{r-1} r\left(h_{i i}^{0 a}\right)^{2}+\frac{r-1}{2}\left(h_{r r}^{0 a}\right)^{2}-4 \sum_{1 \leq i<j \leq r} h_{i i}^{0 a} h_{j j}^{0 a},
\end{aligned}
$$

and:

$$
\begin{aligned}
\varphi_{a}\left(h_{11}^{0 a}, h_{22}^{0 a}, \ldots, h_{k-1, k-1}^{0 a}, h_{k k}^{0 a}\right) \\
\quad=\sum_{l=1}^{k-1} k\left(h_{l l}^{0 a}\right)^{2}+\frac{k-1}{2}\left(h_{k k}^{0 a}\right)^{2}-4 \sum_{1 \leq l<s \leq k} h_{l l}^{0 a} h_{s s}^{0 a} .
\end{aligned}
$$

First, we consider the constrained extremum problem $\min \phi_{a}$ subject to:

$$
Q: h_{11}^{0 a}+\cdots+h_{r r}^{0 a}=t^{a}, \quad\left(t^{a} \text { is any constant }\right) .
$$

From (38), we find that the critical points

$$
h^{0 c}=\left(h_{11}^{0 a}, h_{22}^{0 a}, \ldots, h_{r-1, r-1}^{0 a}, h_{r r}^{0 a}\right)
$$

of $Q$ are the solutions of the following system of linear homogeneous equations.

$$
\left.\begin{array}{c}
\frac{\partial \phi_{a}}{\partial h_{i i}^{0 a}}=2(r+2)\left(h_{i i}^{0 a}\right)-4 \sum_{j=1}^{r} h_{j j}^{0 a}=0, \\
\frac{\partial \phi_{a}}{\partial h_{r r}^{0 a}}=(r-1) h_{r r}^{0 a}-4 \sum_{j=1}^{r-1} h_{j j}^{0 a}=0,
\end{array}\right\}
$$

for $i \in\{1,2, \ldots, r-1\}$ and $a \in\{m+1, \ldots, n\}$. Hence, every solution $h^{0 c}$ has:

$$
h_{i i}^{0 a}=\frac{1}{r+1} t^{a}, \quad h_{r r}^{0 a}=\frac{4}{r+3} t^{a},
$$

for $i \in\{1,2, \ldots, r-1\}$ and $a \in\{m+1, \ldots, n\}$.

Now, we fix $x \in Q$. The bilinear form $\Theta: T_{x} Q \times T_{x} Q \rightarrow \mathbb{R}$ has the following expression (cf. Theorem 1):

$$
\Theta(E, F)=\operatorname{Hess}_{\phi_{a}}(E, F)+\left\langle h^{\prime}(E, F), \operatorname{grad}\left(\phi_{a}\right)(x)\right\rangle,
$$

where $h^{\prime}$ denotes the second fundamental form of $Q$ in $\mathbb{R}^{r}$ and $<\cdot, \cdot>$ denotes the standard inner product on $\mathbb{R}^{r}$. The Hessian matrix of $\phi_{a}$ is given by:

$$
\text { Hess }_{\phi_{a}}=\left(\begin{array}{ccccc}
2(r+2) & -4 & \ldots & -4 & -4 \\
-4 & 2(r+2) & \ldots & -4 & -4 \\
\vdots & \vdots & \ddots & \vdots & \vdots \\
-4 & -4 & \ldots & 2(r+2) & -4 \\
-4 & -4 & \ldots & -4 & (r-1)
\end{array}\right) .
$$


Take a vector $E \in T_{x} Q$, which satisfies a relation $\sum_{i=1}^{r} E_{i}=0$. As the hyperplane is totally geodesic, i.e., $h^{\prime}=0$ in $\mathbb{R}^{r}$, we get:

$$
\begin{aligned}
\Theta(E, E) & =\operatorname{Hess}_{\phi_{a}}(E, E) \\
& =2(r+2) \sum_{i=1}^{r-1} E_{i}^{2}+(r-1) E_{r}^{2}-8 \sum_{i \neq j=1}^{r} E_{i} E_{j} \\
& =2(r+2) \sum_{i=1}^{r-1} E_{i}^{2}+(r-1) E_{r}^{2}-4\left\{\left(\sum_{i=1}^{r} E_{i}\right)^{2}-\sum_{i=1}^{r} E_{i}^{2}\right\} \\
& =2(r+4) \sum_{i=1}^{r-1} E_{i}^{2}+(r+3) E_{r}^{2} \\
& \geq 0 .
\end{aligned}
$$

However, the point $h^{0 c}$ is the only optimal solution, i.e., the global minimum point of problem, and reaches a minimum $Q\left(h^{0 c}\right)=0$ by considering (39) and the constrained extremum problem $\min \varphi_{a}$ subject to:

$$
Q^{\prime}: h_{11}^{0 a}+\cdots+h_{k k}^{0 a}=\alpha^{a}, \quad\left(\alpha^{a} \text { is any constant }\right) .
$$

Thus, we have:

$$
\begin{aligned}
2 \sigma^{\nabla, \nabla^{*}} \leq & r(r-1) \mathcal{C}_{1}^{0}+\left(r^{2}-1\right) \mathcal{C}_{1}^{0}\left(W_{1}\right)+\frac{r}{2}\left(\mathcal{C}_{1}+\mathcal{C}_{1}^{*}\right) \\
& +k(k-1) \mathcal{C}_{2}^{0}+\left(k^{2}-1\right) \mathcal{C}_{2}^{0}\left(W_{2}\right)+\frac{k}{2}\left(\mathcal{C}_{2}+\mathcal{C}_{2}^{*}\right) \\
& +\frac{k \Delta^{N_{1} 0} \mathfrak{f}}{\mathfrak{f}}+r(r-1) \tilde{c}+k(k-1) \tilde{c} \\
& -\frac{r^{2}}{2}\left(\left\|\mathcal{H}_{1}\right\|^{2}+\left\|\mathcal{H}_{1}^{*}\right\|^{2}\right)-\frac{k^{2}}{2}\left(\left\|\mathcal{H}_{2}\right\|^{2}+\left\|\mathcal{H}_{2}^{*}\right\|^{2}\right)
\end{aligned}
$$

Consequently, we get immediately the following theorem from the above relation:

Theorem 6. Let $N=N_{1} \times_{\mathfrak{f}} N_{2}$ be an m-dimensional statistical warped product submanifold immersed into an n-dimensional statistical manifold of constant sectional curvature $\tilde{c}$. Then, the Casorati curvatures satisfy:

$$
\begin{aligned}
2 \sigma^{\nabla, \nabla^{*}} \leq & r(r-1) \mathcal{C}_{1}^{0}+\left(r^{2}-1\right) \mathcal{C}_{1}^{0}\left(W_{1}\right)+r \mathcal{C}_{1}^{0} \\
& +k(k-1) \mathcal{C}_{2}^{0}+\left(k^{2}-1\right) \mathcal{C}_{2}^{0}\left(W_{2}\right)+k \mathcal{C}_{2}^{0} \\
& +\frac{k \Delta^{N_{1} 0_{\mathfrak{f}}}}{\mathfrak{f}}+r(r-1) \tilde{c}+k(k-1) \tilde{c} \\
& -\frac{r^{2}}{2}\left(\left\|\mathcal{H}_{1}\right\|^{2}+\left\|\mathcal{H}_{1}^{*}\right\|^{2}\right)-\frac{k^{2}}{2}\left(\left\|\mathcal{H}_{2}\right\|^{2}+\left\|\mathcal{H}_{2}^{*}\right\|^{2}\right)
\end{aligned}
$$

where $W_{1}$ and $W_{2}$ are respectively the hyperplanes of $T_{p} N_{1}$ and $T_{p} N_{2}, \mathcal{C}_{1}^{0}=\frac{1}{2}\left(\mathcal{C}_{1}+\mathcal{C}_{1}^{*}\right)$, and $\mathcal{C}_{2}^{0}=\frac{1}{2}\left(\mathcal{C}_{2}+\mathcal{C}_{2}^{*}\right)$.

\section{Examples}

We provide examples of statistical warped product submanifolds as follows:

Example 1. By generalizing Example 2.7 of [10] to higher dimensions, we see that:

$$
\left(\mathbb{R} \times e^{z} \mathbb{R}^{n}, \tilde{g}=d z^{2}+e^{2 z}\left(d x_{1}^{2}+\cdots+d x_{n}^{2}\right), \nabla, \nabla^{*}\right)
$$


is a statistical warped product manifold. Furthermore, the hyperbolic space:

$$
\mathbb{H}^{n+1}(-1)=\left(\left\{\left(x_{0}, \ldots, x_{n+1}\right) \in \mathbb{R}^{n+1} \mid x_{0}>0\right\}, \tilde{g}=\frac{d x_{0}^{2}+\cdots+d x_{n+1}^{2}}{x_{0}^{2}}, \tilde{\nabla}, \tilde{\nabla}^{*}\right)
$$

is the statistical manifold of constant sectional curvature -1 . Thus, with respect to the Levi-Civita connection, $\mathbb{R} \times e^{z} \mathbb{R}^{n-1}$ admits an isometric minimal immersion into $\mathbb{H}^{n+1}(-1)$.

Example 2. $\left(\mathbb{R} \times_{z} \mathbb{R}^{n}, \tilde{g}=d t^{2}+t^{2}\left(d x_{1}^{2}+\cdots+d x_{n}^{2}\right), \nabla, \nabla^{*}\right)$ is a statistical warped product manifold, and it is isometric to the Euclidean $(n+1)$-space $\mathbb{E}^{n+1}$. Let $N$ be a minimal submanifold of the unit hypersphere $S^{n}(1) \subset \mathbb{E}^{n+1}$ center at the origin $o \in \mathbb{E}^{n+1}$, and let $C(N)$ be the cone over $N$ with the vertex at 0 .

The metric of $C(N)$ is the warped product metric $g_{C(N)}=d t^{2}+t^{2} g_{N}$, where $g_{N}$ denotes the metric of $N$. Any open submanifold $M$ of $C(N)$ is a warped product manifold, which admits an isometric minimal immersion into the statistical manifold $\mathbb{E}^{n+1}$ of constant sectional curvature zero.

Author Contributions: Conceptualization, A.N.S., B.-Y.C. and O.B.; Methodology, A.N.S., B.-Y.C. and O.B.; Software, A.N.S. and B.-Y.C.; Validation, A.N.S., B.-Y.C. and O.B.; Formal Analysis, A.N.S.; Investigation, A.N.S., B.-Y.C. and O.B.; Resources, A.N.S.; Data curation, A.N.S. and B.-Y.C.; Writing-Original Draft Preparation, A.N.S.; Writing-Review and Editing, A.N.S. and B.-Y.C.; Visualization, A.N.S.; Supervision, B.-Y.C.; Project Administration, A.N.S., B.-Y.C. and O.B.; Funding Acquisition, A.N.S., B.-Y.C. and O.B.

Funding: This research received no external funding.

Acknowledgments: The authors thank the referees for many valuable and helpful suggestions to improve the presentation of this article.

Conflicts of Interest: The authors declare no conflict of interest.

\section{References}

1. Amari, S. Differential-Geometrical Methods in Statistics; Lecture Notes in Statistics; Springer: New York, NY, USA, 1985; Volume 28.

2. Lauritzen, S. Statistical manifolds. In Differential Geometry in Statistical Inference; Amari, S.I., Barndorff-Nielsen, O.E., Kass, R.E., Lauritzen, S.L., Rao, C.R., Eds.; IMS Lecture Notes Institute of Mathematical Statistics: Hayward, CA, USA, 1987; Volume 10, pp. 163-216.

3. Bishop, R.L.; O'Neill, B. Manifolds of negative curvature. Trans. Am. Math. Soc. 1969, 145, 1-49. [CrossRef]

4. Chen, B.-Y. Pseudo-Riemannian Geometry, S-Invariants and Applications; Worlds Scientific: Hackensack, NJ, USA, 2011.

5. Chen, B.-Y. Differential Geometry of Warped Product Manifolds and Submanifolds; Worlds Scientific: Hackensack, NJ, USA, 2017.

6. Todjihounde, L. Dualistic structures on warped product manifolds. Differ. Geom.-Dyn. Syst. 2006, 8, $278-284$.

7. Furuhata, H.; Hasegawa, I.; Okuyama, Y.; Sato, K. Kenmotsu statistical manifolds and warped product. J. Geom. 2017, 108, 1175-1191. [CrossRef]

8. Furuhata, H. Hypersurfaces in statistical manifolds. Differ. Geom. Appl. 2009, 27, 420-429. [CrossRef]

9. Aytimur, H.; Ozgur, C. Einstein statistical warped product manifolds. Filomat 2018, 32, 3891-3897. [CrossRef]

10. Murathan, C.; Sahin, B. A study of Wintgen like inequality for submanifolds in statistical warped product manifolds. J. Geom. 2018, 109, 30. [CrossRef]

11. Chen, B.-Y. On isometric minimal immersions from warped products into real space forms. Proc. Edinb. Math. Soc. 2002, 45, 579-587. [CrossRef]

12. Chen, B.-Y. Warped products in real space forms. Rocky Mt. J. Math. 2004, 34, 551-563. [CrossRef]

13. Casorati, F. Mesure de la courbure des surfaces suivant l'idée commune. Acta Math. 1890, 14, 95-110. [CrossRef]

14. Lee, C.W.; Lee, J.W.; Vilcu, G.E.; Yoon, D.W. Optimal inequalities for the Casorati curvatures of the submanifolds of generalized space form endowed with semi-symmetric metric connections. Bull. Korean Math. Soc. 2015, 52, 1631-1647. [CrossRef] 
15. Lee, C.W.; Vilcu, G.E. Inequalities for generalized normalized Casorati curvatures of slant submanifolds in quaternion space forms. Taiwan. J. Math. 2015, 19, 691-702. [CrossRef]

16. Lee, C.W.; Lee, J.W.; Vilcu, G.E. Optimal inequalities for the normalized $\delta$-Casorati curvatures of submanifolds in Kenmotsu space forms. Adv. Geom. 2017, 17, 355-362. [CrossRef]

17. Shahid, M.H.; Siddiqui, A.N. Optimizations on totally real submanifolds of LCS-manifolds using Casorati curvatures. Commun. Korean Math. Soc. 2019, 34, 603-614.

18. Siddiqui, A.N.; Shahid, M.H. A lower bound of normalized scalar curvature for bi-slant submanifolds in generalized Sasakian space forms using Casorati curvatures. Acta Math. Univ. Comen. 2018, 87, 127-140.

19. Siddiqui, A.N. Upper bound inequalities for $\delta$-Casorati curvatures of submanifolds in generalized Sasakian space forms admitting a semi-Symmetric metric connection. Int. Electron. J. Geom. 2018, 11, 57-67.

20. Siddiqui, A.N. Optimal Casorati inequalities on bi-slant submanifolds of generalized Sasakian space forms. Tamkang J. Math. 2018, 49, 245-255. [CrossRef]

21. Slesar, V.; Sahin, B.; Vilcu, G.E. Inequalities for the Casorati curvatures of slant submanifolds in quaternionic space forms. J. Inequal. Appl. 2014, 2014, 123. [CrossRef]

22. Aydin, M. E.; Mihai, I. Wintgen inequality for statistical surfaces. Math. Inequal. Appl. 2019, 22, $123-132$. [CrossRef]

23. Alkhaldi, A.H.; Aquib, M.; Siddiqui, A.N.; Shahid, M.H. Pinching theorems for statistical submanifolds in Sasaki-like statistical space forms. Entropy 2018, 20, 690. [CrossRef]

24. Decu, S.; Haesen, S.; Verstraelen, L.; Vilcu, G.E. Curvature invariants of statistical submanifolds in Kenmotsu statistical manifolds of constant $\phi$-sectional curvature. Entropy 2018, 20, 529. [CrossRef]

25. Lee, C.W.; Yoon, D.W.; Lee, J.W. A pinching theorem for statistical manifolds with Casorati curvatures. J. Nonlinear Sci. Appl. 2017, 10, 4908-4914. [CrossRef]

26. Siddiqui, A.N.; Shahid, M.H. Optimizations on statistical hypersurfaces with Casorati curvatures. Kragujevac J. Math. 2021, 45, 449-463.

27. Yano, K.; Kon, M. Structures on Manifolds; Worlds Scientific: Singapore, 1984.

28. Vos, P.W. Fundamental equations for statistical submanifolds with applications to the Bartlett correction. Ann. Inst. Stat. Math. 1989, 41, 429-450. [CrossRef]

29. Opozda, B. Bochner's technique for statistical structures. Ann. Glob. Anal. Geom. 2015, 48, 357-395. [CrossRef]

30. Opozda, B. A sectional curvature for statistical structures. Linear Algebra Appl. 2016, 497, 134-161. [CrossRef]

31. Oprea, T. Optimization methods on Riemannian submanifolds. Analele Univ. Buc. 2005, 54, 127-136.

32. Hamilton, R.S. The Ricci flow on surfaces. Contemp. Math. 1988, 71, 237-261.

33. Chen, B.-Y. Ricci solitons on Riemannian submanifolds. In Riemannian Geometry and Applications-Proceedings RIGA; University of Bucharest Press: Bucharest, Romania, 2014; pp. 30-45.

34. Mantica, C.A.; Shenawy, S.; Unal, B. Ricci solitons on singly warped product manifolds and applications. arXiv 2019, arXiv:1508.02794v2.

35. Meric, S.E.; Kilic, E. Some inequalities for Ricci solitons. In Proceedings of International Conference on Mathematics and Mathematics Education (ICMME 2018), Ordu, Turkey, 27-30 June 2018; Volume 10, pp. 160-164.

36. Crasmareanu, M. A new approach to gradient Ricci solitons and generalizations. Filomat 2018, 32, 3337-3346. [CrossRef]

37. Calin, O.; Udriste, C. Geometric Modeling in Probability and Statistics; Springer: Cham, Switzerland, 2014.

38. Chen, B.-Y. Some pinching and classification theorems for minimal submanifolds. Arch. Math. 1993, 60, 568-578. [CrossRef]

39. Oprea, T. On a Riemannian invariant of Chen type. Rocky Mountain J. Math. 2008, 38, 567-581. [CrossRef]

(C) 2019 by the authors. Licensee MDPI, Basel, Switzerland. This article is an open access article distributed under the terms and conditions of the Creative Commons Attribution (CC BY) license (http:/ / creativecommons.org/licenses/by/4.0/). 